were observed to have extravasated after $60 \mathrm{~min}$ (Abstract 119 figure 2 ). Coronary flow was not affected by injection of BMMNCs, whereas MSC injection led to a transient decrease of coronary flow.
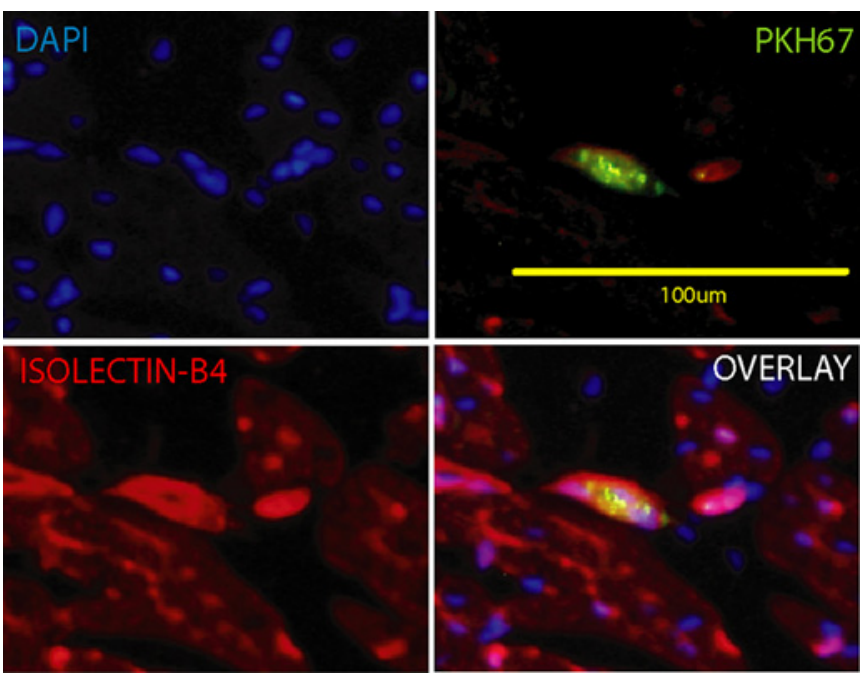

Abstract 119 Figure 2

Conclusion Our study using an original model showed retention rates of BMMNC in normal hearts were low regardless of the cell number injected, suggesting a critical limiting factor for the treatment's success. Retention rates were improved by I-R. Larger BMMNCs were retained with an increased efficiency. This information provides intriguing potential methods for increasing retention in future clinical studies, thereby enhancing the therapeutic effect.

\section{BLOOD FLOW IS REQUIRED FOR ENDOTHELIAL REPAIR IN A NOVEL IN VIVO MODEL USING ZEBRAFISH EMBRYOS}

doi:10.1136/heartjnl-2012-301877b.120

N M Quaife,* T J A Chico. MRC Centre for Developmental and Biomedical Genetics, University of Sheffield, Sheffield, UK

Introduction Endothelial repair is essential for vascular homeostasis but difficult to study in vivo using existing animal models. The role of blood flow in the repair response remains unclear. We therefore established a novel in vivo model of endothelial repair using transgenic zebrafish embryos, and tested whether this was affected by the absence of blood flow.

Methods 4-day old Fli1:eGFP transgenic embryos (expressing green fluorescent protein in endothelium) were anaesthetised and mounted in low melting point agarose. We used focused laser injury (Micropoint laser mounted on a Nikon inverted microscope) to ablate the endothelium of the dorsal aorta in the region between somites 15-17. Time-lapse fluorescence or confocal microscopy was then used to visualise recovery of the ablated endothelium in real time. To determine the role of blood flow in endothelial repair, embryos were treated with the anaesthetic tricaine which reversibly halts cardiac contraction and recovery from aortic ablation determined as above. This does not affect the embryos' development, which survive due to oxygen diffusion.

Results Abstract 120 figure 1 demonstrates endothelial ablation at $0 \mathrm{~h}$. Over the following $14.5 \mathrm{~h}$ we observed migration of endothelial cells from both proximal and distal vasculature (aorta, vein, and intersegmental vessels) to repopulate the ablated region, suggesting this is a model of endothelial migration and proliferation. We did not observe recruitment of circulating cells to the site of injury. Complete recovery in all ablated embryos was achieved by $15-20 \mathrm{~h}$ post injury. Reversible cessation of blood flow significantly prevented endothelial repair compared with control (fluorescence at
$20 \mathrm{~h}$ post injury: control $60 \% \pm 22 \%$ of baseline vs absent blood flow $5 \% \pm 2.4 \%, \mathrm{p}<0.05$ (Abstract 120 figure 2).

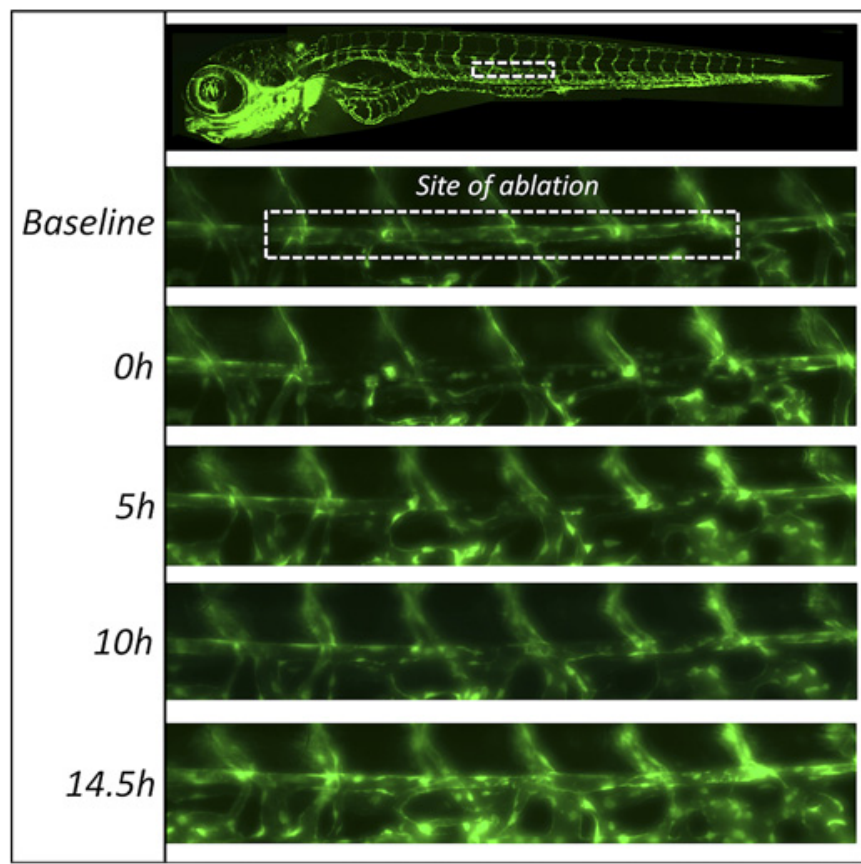

Abstract 120 Figure 1 Timecourse of endothelial repair following laser injury.

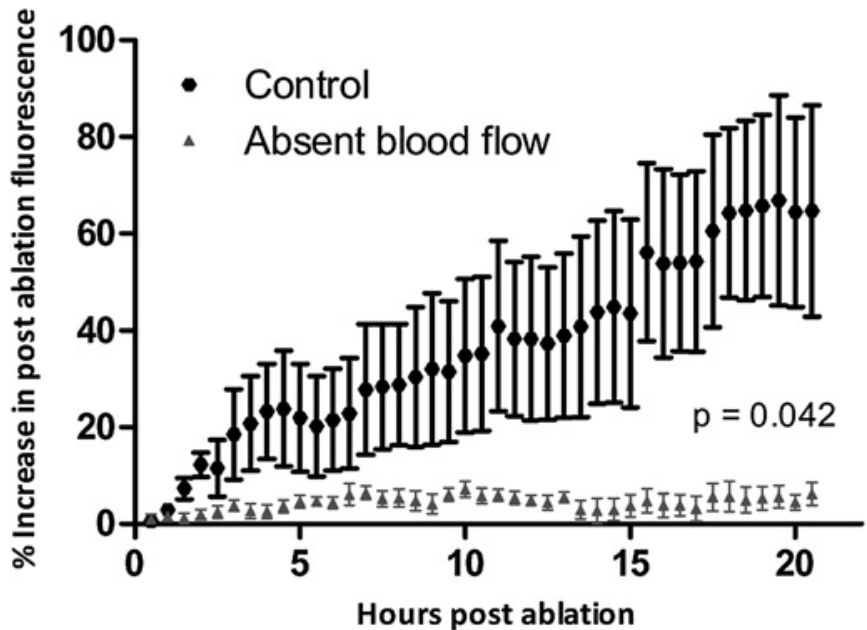

Abstract 120 Figure 2 Timecourse of endothelial repair in embryos with (black) and without (grey) blood flow.

Conclusions The ability to visualise endothelial damage and recovery in living zebrafish embryos offers a powerful new model for dissecting the mechanisms of endothelial repair in vivo. Endothelial repair requires blood flow even in the absence of recruitment of circulating cells.

\section{BIOINFORMATIC IMAGING AND MOLECULAR INVESTIGATION FOR A ROLE OF P22 ${ }^{\text {PHOX }}$ C242T POLYMORPHISM IN INHIBITING ENDOTHELIAL ROS PRODUCTION}

doi:10.1136/heartjnl-2012-301877b.121

D N Meijles, * B J Howlin, J-M Li. University of Surrey, Surrey, UK

The $\mathrm{p} 22^{\text {phox }}$ is a key component of the cytochrome b558 of the NADPH oxidase (Nox), which by generating reactive oxygen species 
(ROS) is involved in the pathogenesis of many cardiovascular diseases. Several clinical studies have reported that a single nucleotide polymorphism (SNP) of p22 $2^{\text {phox }}(\mathrm{C} 242 \mathrm{~T})$ is negatively associated with the incidence of coronary heart disease. However, the mechanism involved remains unknown. In this study we combined computer molecular modelling with cell and molecular biology to investigate the potential mechanism of the p22 phox $C 242 \mathrm{~T}$ on endothelial ROS production. Our in silico $3 \mathrm{D}$ protein structure model of $\mathrm{p} 22^{\text {phox }}$ consists of an N-terminal transmembrane domain (124 a.a.) and a C-terminal cytoplasmic domain (71 a.a.). The p22 $2^{\text {phox }}$ C242T causes a substitution of His72 to Tyr72 in its extracellular loop, which results in significant morphological changes in the putative interactive region of the $\mathrm{p} 22^{\text {phox }}$ with the catalytic subunit (Nox2), and this may result in a reduced ROS production. To test this hypothesis, we generated $\mathrm{p} 22^{\text {phox }} \mathrm{C} 242 \mathrm{~T}$ construct by site-directed mutagenesis and transfected mouse microvascular endothelial cells (SVEC4-10) with either wild-type (WT) $2_{2} 2^{\text {phox }}$ or p22 $2^{\text {phox }}$ C242T. Compared to WT control, C242T had no significant effect on basal ROS production. However, when cells were stimulated with TNF $\alpha$ (100 U/ml, $45 \mathrm{~min})$, C242T significantly reduced $(48 \% \pm 5.6 \%)$ the levels of ROS production detected by both lucigenin-chemiluminescence and DCF fluorescence. Reduced ROS production was accompanied with inhibition in TNF $\alpha$-induced ERK1/2 and p38MAPK activation and NFKB phosphorylation. Furthermore, we found that C242T reduced significantly $(31.9 \% \pm 2 \%)$ the TNF $\alpha(12 \mathrm{~h})$-induced endothelial cell apoptosis as detected by Annexin V flow cytometry. In conclusion, we reported for the first time that the p22 $2^{\text {phox }} \mathrm{C} 242 \mathrm{~T}$ SNP is associated with a significant protein morphological change, which affects the activity of NADPH oxidase, and reduces the ROS production by endothelial cells when challenged by TNF $\alpha$. Our study provides important insight into the mechanism of $p 22^{\text {phox }}$ C242T SNP and its clinical relevance as a natural inhibitory factor of the incidence of coronary heart disease (CHD).

\section{AN INTERVENTION TO ALTER HELP-SEEKING BEHAVIOUR AND REDUCE PRE-HOSPITAL DELAY TIME IN PATIENTS DIAGNOSED WITH ACUTE CORONARY SYNDROME (ACS)}

doi:10.1136/heartjnl-2012-301877b.122

${ }^{1} \mathrm{M}$ Mooney, ${ }^{*}{ }^{1} \mathrm{~F}$ O'Brien, ${ }^{1} \mathrm{G}$ McKee, ${ }^{1} \mathrm{~S}$ O' Donnell, ${ }^{2} \mathrm{D}$ Moser. ${ }^{1}$ Trinity College Dublin, Dublin, Ireland; ' University of Kentucky, Lexington, Kentucky, USA

Introduction The problem of pre-hospital delay among patients with acute coronary syndrome remains unresolved despite past attempts to contain the problem. Mortality and morbidity could be reduced if patients sought earlier treatment for symptoms. Median prehospital delay times ranging from $1.0 \mathrm{~h}$ to $15.2 \mathrm{~h}$ have been reported in the literature. Most of the reported pre-hospital delay times extend beyond the parameters outlined in the European Society of Cardiology guidelines.

Methods A randomised controlled trial was used to test the effectiveness of an educational intervention on pre-hospital delay time and help-seeking behaviours including contacting the general practitioner, use of nitrates, use of ambulance and time taken to notify someone in response to ACS symptoms. The intervention group were given a 30 -min individualised education session which focused on: education about heart disease misconceptions, stimulating behavioural change through motivational techniques and the provision of a tailored action plan for future use. This intervention was reinforced 1 month later by telephone.

- Operational definition: Pre-hospital delay time was defined as the time from acute symptom onset until arrival at the emergency department.

- Research instrument: Response to Symptoms Questionnaire.
Results Pre-hospital delay time was initially recorded on 1944 patients (control: 972; intervention: 972). Of these, 314 patients were readmitted to an emergency department with ACS symptoms (control: 137; intervention: 177). We again measured their prehospital delay times. The readmission diagnoses were: control group: (STEMI: 24.8\%; NSTEMI 25.5\%, unstable angina $49.6 \%$ ) and intervention group: (STEMI 24.3\%; NSTEMI 35.0\%; unstable angina $40.7 \%)$. Data were collected using patient interviews and prehospital delay times were subsequently verified from the case notes. Allowing for the covariate of education, on which the two groups differed, we used repeated measures ANCOVA on log transformed delay-time. Those in the intervention group demonstrated a significant decrease in pre-hospital delay time compared to both their own baseline $(p<0.001)$ and to that of the control group $(p=0.04)$. The intervention group also had a lower rate of consultation with a general physician before attending the emergency department $(p=0.024)$ and were quicker to report the presence of symptoms to another individual $(p=0.010)$. The groups did not differ on the use of ambulance $(p=0.510)$ or nitrates $(p=0.058)$.

Conclusions/Implications This RCT demonstrated that it is possible to reduce pre-hospital delay time in patients diagnosed with ACS. This can be achieved through an individualised educational intervention that focuses on symptom identification and the appropriate actions to take in this situation. There is a need for renewed and continued emphasis on the importance of ambulance use when ACS symptoms arise.

\section{IRISH PATIENTS' PERCEPTIONS OF THEIR RISK FOR A HEART ATTACK IN THE FUTURE FOLLOWING THE DIAGNOSIS OF ACUTE CORONARY SYNDROME}

doi:10.1136/heartjnl-2012-301877b.123

${ }^{1} \mathrm{~F}$ O'Brien, ${ }^{* 1} \mathrm{M}$ Mooney, ${ }^{1} \mathrm{~S}$ O'Donnell, ${ }^{1} \mathrm{G}$ McKee, ${ }^{2} \mathrm{D}$ Moser. ${ }^{1}$ Trinity College Dublin, Dublin, Ireland; ' ${ }^{2}$ University of Kentucky, Lexington, Kentucky, USA

Introduction People who perceive themselves to be at risk of an acute coronary syndrome (ACS) are more likely to seek treatment when ACS symptoms arise. This in turn leads to appropriate treatmentseeking behaviour and earlier medical intervention, which are crucial to successful outcomes. However, many people fail to appreciate their true risk of a future heart attack and are thus less likely to seek timely treatment for ACS symptoms. Consequently, their risk of mortality and morbidity is increased relative to those who perceive themselves to be at risk. The aims of this study are to ascertain how individuals who have been diagnosed with ACS perceive their future risk of heart attack and to identify the differentiating characteristics associated with perceptions of such risk.

Methods Data were collected from 1946 patients admitted to hospital with an ACS event. Recruitment took place across five of the major acute hospitals in Dublin, Ireland. Ethical approval was granted for this cross-sectional study. The ACS Response Index was the research instrument used for this study. As a component of this, patients were asked the following question: "Compared to other people your age, how likely do you think it is that you could have a heart attack in the next 5 years?" Data were analysed using PASW v18.

Results The total sample mean age was 63 years \pm 11.7 years, $72 \%$ were male. Patient diagnoses comprised of: STEMI (28\%), NSTEMI $(36.4 \%)$ and unstable angina (35.6\%). A total of 732 (37.6\%) felt that their risk was about the same as others their age, while $359(18.5 \%)$ felt that their risk was lower. The remaining 855 (43.9\%) felt their risk was higher than others their age. To determine the characteristics of those who accurately assessed their risk (felt that relative to others their own age, it was at least equivalent or higher) we performed logistic regression using the following variables: age, 